\title{
Pulse and Vital Sign Measurement in Mixed Reality using a HoloLens
}

\author{
Daniel McDuff \\ Microsoft Research \\ Redmond, USA \\ damcduff@microsoft.com
}

\author{
Christophe Hurter \\ Ecole Nationale de l'Aviation Civile \\ Toulouse, France \\ christophe.hurter@enac.fr
}

\author{
Mar Gonzalez-Franco \\ Microsoft Research \\ Redmond, USA \\ margon@microsoft.com
}

\begin{abstract}
Cardiography, quantitative measurement of the functioning of the heart, traditionally requires customized obtrusive contact sensors. Using new methods photoplethysmography and ballistocardiography signals can be captured using ubiquitous sensors, such as webcams and accelerometers. However, these signals are not visible to the unaided eye. We present Cardiolens - a mixed reality system that enables real-time, hands-free measurement and visualization of blood flow and vital signs from multiple people. The system combines a front-facing webcam, imaging ballistocardiography, and remote imaging photoplethysmography methods for recovering pulse signals. A heads up display allows users to view their own heart rate whenever they are wearing the device and the heart rate and heart rate variability of another person simply by looking at them. Cardiolens provides the wearer with a new way to understand physiological signals and has applications in human-computer interaction and in the study of social psychology.
\end{abstract}

\section{CCS CONCEPTS}

-Human-centered computing $\rightarrow$ Mixed / augmented reality; Visualization; $\cdot$ Computing methodologies $\rightarrow$ Biometrics;

\section{KEYWORDS}

Mixed reality; remote sensing; physiology; interoception; health.

\section{INTRODUCTION}

Analyzing physiological and emotional states is important for understanding health and well-being, memory, decision-making, as well as enabling more meaningful social interactions. Convenient, unobtrusive measurement of these signals has many applications. Vital signs are important basic health parameters and simultaneously provide information about a person's affective state. When
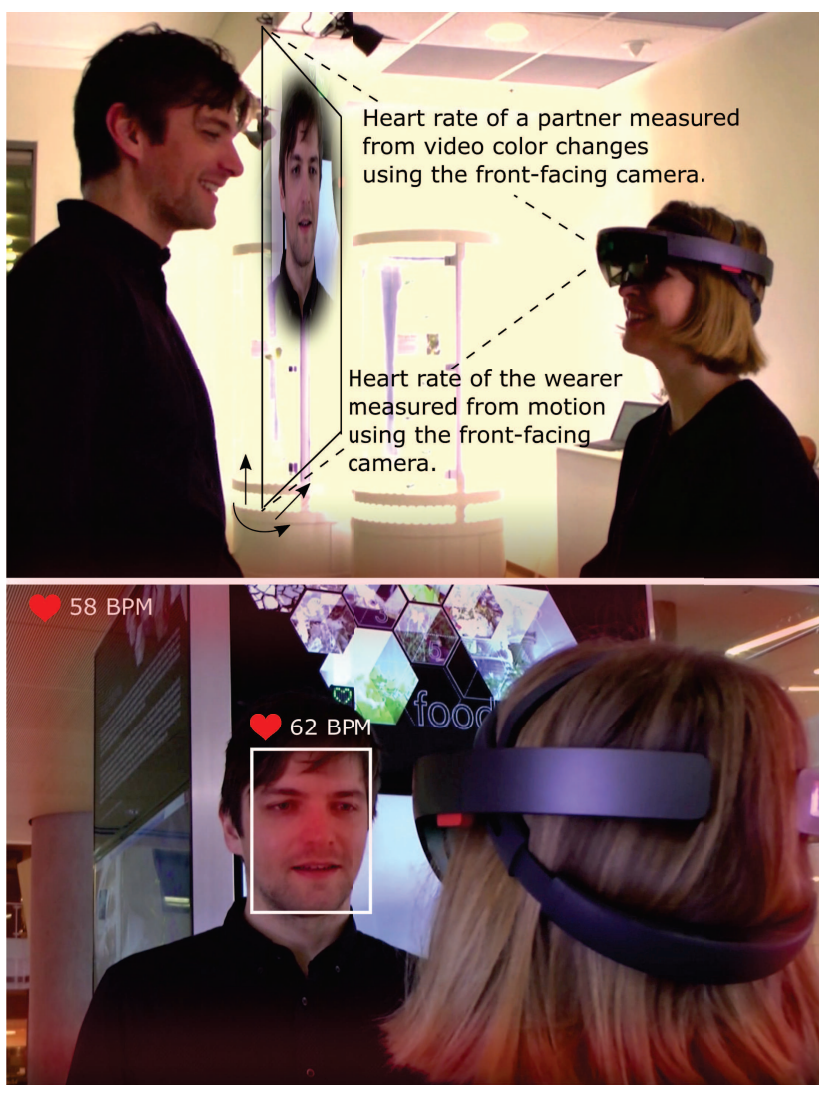

Figure 1: Cardiolens is a mixed reality system that allows simultaneous physiological measurement of multiple people, including the wearer. The physiological signals are visualized in the real-world allowing the wearer to see magnified blood perfusion and vital signs in real-time.

rendered in real-time these signals may be powerful tools for interoceptive awareness (i.e. the consciousness of our physiological and inner bodily state, which relates to the experience of emotions [Bechara and Naqvi 2004]) and synchronization. We present Cardiolens - a mixed reality system that enables real-time, hands free measurement of blood flow and vital signs of multiple people (including the wearer) concomitantly. Cardiolens presents new possibilities for immersive experiences, augmented social interactions and as a tool for practitioners in several contexts such as healthcare and personal training. Figure 1 shows an overview of the system. 


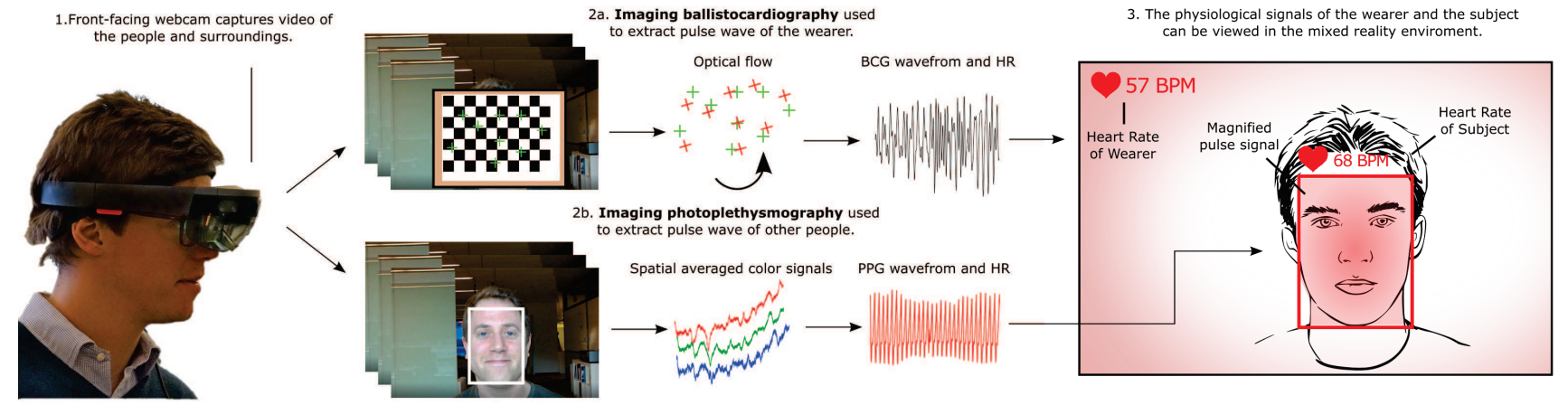

Figure 2: Schematic of the Cardiolens system. Images from the front-facing camera are used to extract the physiological state of the wearer and the person they are looking at. Imaging photoplethysmography is used to recover the pulse wave of the person being looked at. Imaging ballistocardiography is used to recover the pulse wave of the wearer. These signals are used to augment the appearance of the real-world allowing the wearer to see the normally "hidden" physiological information in real-time.

Cardiolens leverages the embedded camera of the mixed reality device and two forms of cardiography for recovering physiological measurements from the person wearing the device (from now on referred to as the wearer) and a person they are looking at (from now on referred to as the partner) simultaneously. The first form of cardiography is based on photoplethysmography, a method of capturing the blood volume pulse (BVP) via small variations in light reflected from the skin [Allen 2007]. Remote imaging photoplethysmography (iPPG) is an advanced set of computer vision methods that enables PPG measurement using just a webcam and ambient light [Poh et al. 2010, 2011]. A survey of these techniques can be found in [McDuff et al. 2015]. We utilize iPPG to retrieve the pulse signal of the partner.

The second form of cardiography is based on ballistocardiography, a technique for sensing heart functioning via minute motions of the body due to the mechanical movements resulting from blood flow [Starr et al. 1939]. We use imaging ballistocardiography (iBCG) as a complementary set of video processing methods for capturing the physiological signals of the wearer. As with iPPG, iBCG measurements can also be obtained from webcam videos. This is possible because the device is head-mounted to the wearer [Hernandez et al. 2014]. Ballistic and inertial techniques can also be used to retrieve the physiological state from accelerometer and gyroscope sensors [Da He et al. 2012; Hernandez et al. 2015]; however, in this work we use an entirely vision based system. As such, it is generalizable to any head worn camera apparatus.

In both iPPG and iBCG analyses the heart rate of an individual can be recovered from the pulse waveforms (see Figure 2). Other information, such as breathing rate or heart rate variability can also be extracted. Preliminary work has shown that these remotely measured physiological signals can be used for contact-free prediction of more complex states, such as whether an individual is under cognitive load [McDuff et al. 2016]. Heart (or Pulse) Rate Variability (HRV) is a useful non-invasive measure of phenomena such as the cardiac regulatory system response, anxiety, or cognitive stress. Being able to measure and visualize this signal from another individual at distance has numerous applications. We discuss the potential use cases and ethical considerations in the Discussion section. This is the first work to our knowledge to attempt to measure HRV from a head mounted device. Furthermore, our results show that HRV time and frequency domain information can be detected.

For the first time, Cardiolens combines iPPG and iBCG measurements from a head-mounted camera to calculate the heart rate, not only of the partner but also of the wearer. Furthermore, this is the first mixed-reality system allowing the wearer to see these physiological signals, such as pulse rate and pulse rate variability, in real-time, in the real-world.

In this regard, new technology can not only provide new ways to measure physiological signals, but can also change how one visualizes or interacts with them. Augmented/mixed reality provides the ability to overlay information onto the real-world. The system brings information from unobtrusive physiological sensing into the user's perceived real-world experience. This can help users associate physiological information with specific physical articles and events.

The unaided human eye does not have the acuity to notice changes in blood perfusion, let alone understand how this physiological phenomenon is changing over time. Therefore, methods for post-processing videos to magnify the subtle changes due to the pulse have been proposed [Wu et al. 2012]. However, many of these magnification approaches do not work in real- or near-real time. Additionally, video magnification does not provide an intuitive real-world visualization of the signals and requires an individual to look at a screen rather than the person, or body part, of interest. New head-mounted wearable devices (e.g., Microsoft HoloLens ${ }^{1}$ or Meta $2^{2}$ ) that feature transparent displays allow dynamic digital information to be overlaid on the real-world and present new opportunities for physiological visualization.

\section{RELATED WORK}

Over the past 10 years there have been considerable advances in the performance of unobtrusive physiological sensing methods [Poh

\footnotetext{
${ }^{1}$ https://www.microsoft.com/microsoft-hololens

${ }^{2}$ https://www.metavision.com
} 


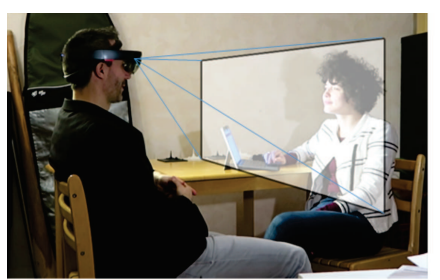

Video Capture

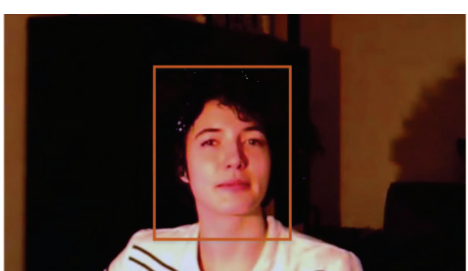

Face Detection

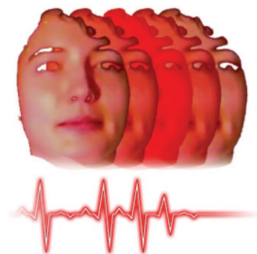

Skin Modification

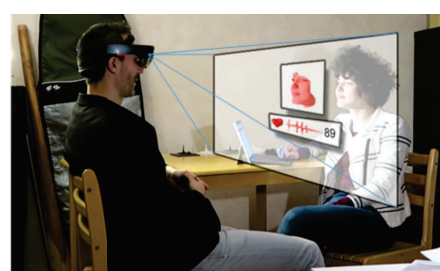

Holographic Composition

Figure 3: Video transformation pipeline for analyzing the physiological signals of another person. The video is captured and processed to extract the subject's heart beat from the facial region-of-interest. Then the system magnifies minute variations in blood flow via modifications to the skin pixel intensities. Finally, holograms are anchored to display the magnified blood volume pulse and heart rate information.

et al. 2010, 2011; Shao et al. 2014; Wang et al. 2015; Zhang et al. 2017]. These methods have been shown to be robust in the presence of head motions [Wang et al. 2015] and to be able to detect subtle aspects of the pulse waveform morphology [McDuff et al. 2014].

Fernando et al. [Fernando et al. 2015] validated remote physiological measurement in neonates using a Google Glass device. The camera on the device was used to recover the PPG waveform and infants' average heart rates. However, no visualization of this signal was proposed and validation of heart rate variability measures were not provided. Google Glass also presented limitations including a very constrained field-of-view (FOV) in the heads-up display and limited processing power. With Cardiolens, we overcome those problems and present a novel mobile system that allows users to view "hidden" physiological signals overlaid in real-time via a semi-transparent mask augmenting the skin color.

Visualization of the blood perfusion signal in videos is possible via Eulerian Video Magnification (EVM) [Wu et al. 2012]. Recently, less computationally expensive versions of these video magnification approaches have been proposed [Wadhwa et al. 2014]. These methods attempt to magnify specific signals within videos (i.e. color change or motion of the face/skin due to the pulse) without effecting other parts of the video (i.e. color or motion in the background). However, inevitably some artifacts are generated. Therefore, augmenting the real-world with physiological signals has some advantages as holograms can be displayed in specific locations without interfering with other elements.

\section{CARDIOLENS}

\subsection{Image Processing Pipeline}

Cardiolens combines iPPG and iBCG methods for recovering physiological measurements from the wearer and the partner simultaneously. In both cases the features are extracted from the front-facing camera images of the Hololens head mounted device. The Hololens has a front-facing, high-definition (1216x684 pixel) camera. We describe the physiological measurement methods below and include results of the validation in the following section. Figure 2 shows the approach graphically.

3.1.1 Imaging Photoplethysmography. A face detector [Viola and Jones 2001] is used to identify the largest face within the images from the front-facing camera. A skin pixel segmentation is applied to the pixels in the facial region of interest. A spacial average of the color channel values for all the skin pixels is calculated. This leads to three time series signals $\mathrm{x}_{1}, \mathrm{x}_{2}, \mathrm{x}_{3}$. In the Validation section we report results for time windows of 20,30 and 60 seconds. We tried preprocessing these signals with the method proposed by Wang et al. [Wang et al. 2017], however, we did not find it consistently improved the results on our dataset and thus we did not employ this method in the remainder of our analysis.

Next, we apply the method proposed by Poh et al. [Poh et al. 2010] to recover the final BVP waveform. Underlying source signals are calculated using Independent Component Analysis (ICA) and then normalized so that they have equal total power in the frequency domain. The BVP estimate is selected as the source signal with greatest peak power in the range $45 \mathrm{BPM}(0.75 \mathrm{~Hz})$ and $180 \mathrm{BPM}(3$ $\mathrm{Hz}$ ).

The Hololens presents a unique challenge not experienced with other cameras. First, as the device is head mounted this results in camera motion that compounds subject motion. Second, the device has a set of powerful infra-red LEDs used for depth and gesture sensing. When these flash they can alter the illumination of the face, this leads to irregular peaks in the video color channel signals. Despite these challenges our results show good performance in heart rate measurement and promising results for heart rate variability measurement.

3.1.2 Imaging Ballistocardiography. To detect the BCG signal of the Cardiolens wearer we apply the Lucas-Kanade optical flow method to the camera images. Using this approach we compute the optical flow between subsequent frames. Lucas-Kanade assumes a constant velocity of subsections of the images and involves a weighted least-square fit of the image derivatives. The resulting frame-to-frame orientation (angular velocity) measurements computed from the $\mathrm{x}$ - and $\mathrm{y}$-velocities are concatenated for a time window. Due to the sensitive nature of the iBCG measurements, capturing subtle changes in head orientation, and the fact that the wearer is likely to have the device on for several minutes at a time we use a 60 second time window to recover the heart rate of the wearer.

\subsection{Physiological Parameter Estimation}

In both the iPPG and iBCG analysis we extract the heart rate as the frequency of the highest peak within the fast Fourier transform (FFT) frequency spectra of the pulse waveform between 45 BPM 
$(0.75 \mathrm{~Hz})$ and $180 \mathrm{BPM}(3 \mathrm{~Hz})$. These values reflect conservative bounds on natural heart rates. The window size for analysis can be calibrated for specific applications. A sliding window means the value can be updated frequently after the initial windows - as frequently as once every frame.

\subsection{Mixed Reality Implementation}

We designed a mixed-reality visualization for Cardiolens, such that the wearer can observe the measured physiological signals in the real-world. This visualization has two components as we measure both the physiological state of the wearer and a partner.

We augment the appearance of the partner with the blood flow signal by altering the brightness of the skin on their face. This process presents the technical challenge of displaying a semi-transparent mask on a moving object to create a mixed reality experience. We developed a holographic overlay which shows the BVP signal superimposed onto the face using a linear image processing pipeline (see Figure 3).

We first turn every captured image from the NV12 (Y, U (Cb) and $\mathrm{V}(\mathrm{Cr})$ pixel packing method) pixel format to the standard red, green and blue (RGB) pixel format. This process is performed using a compute shader (implemented on the GPU) to ensure a sufficient frame rate for real-time performance. Next, the RGB stream is analyzed to extract detected faces. We only consider the largest detected face and we segment skin pixels from the Hololens camera. This is the point at which we perform the iPPG analysis (see the previous section for details). We modify the red channel values of the skin pixels over time to magnify the estimated pulse signal. Finally, we compute the position of the captured frame in the 3D environment. We place this image in the mixed reality space at the average distance of the user from the device (approximately one-meter). We also define a speed vector for this plane, which corresponds to its change of location when the user or subject moves his or her head. The speed estimation is very important to ensure that the overlaid hologram remains stationary on top of the subject's face from the perspective of the Cardiolens wearer. The resulting experience allows the user to see the pulsating blood flow signal that would otherwise be imperceptible. In addition, the heart

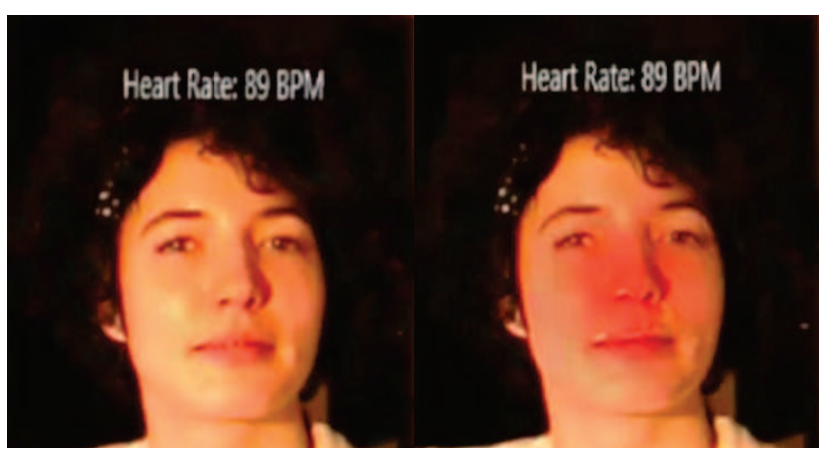

Figure 4: Screenshots of the Cardiolens blood flow augmentation captured during an interaction. The system magnifies minute variations in blood flow captured via the frontfacing camera.
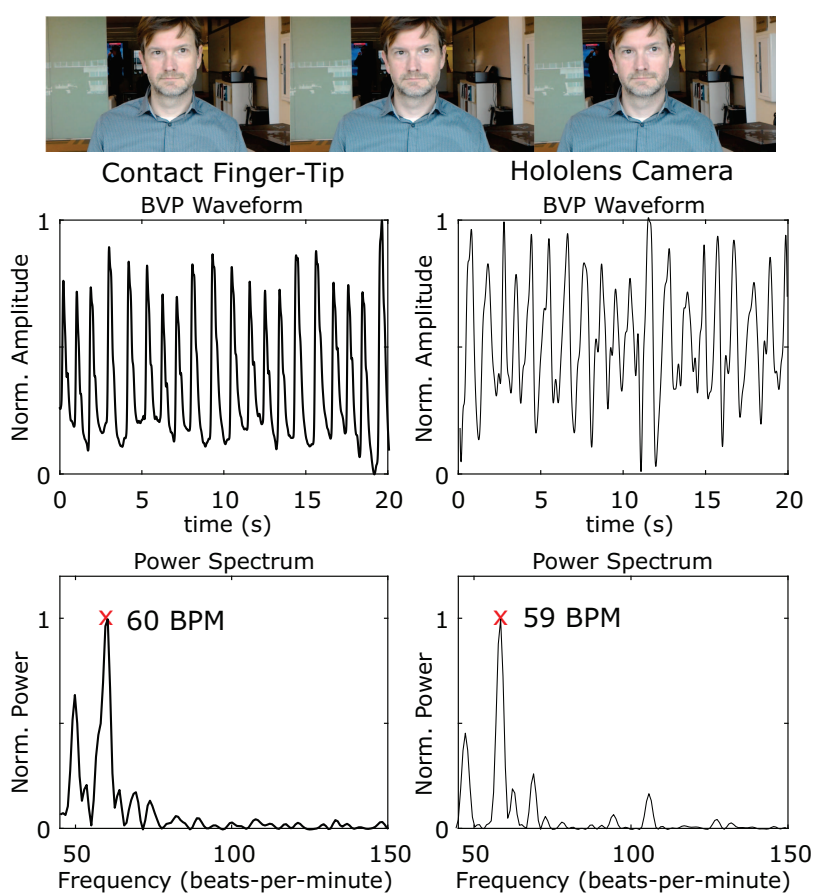

Figure 5: Examples of pulse waveforms from the finger-tip sensor and iPPG measurements. Top) The PPG waveforms. Bottom) The power spectra of the BVP signal and the detected HR. Thumbnail example frames from the Hololens video capture are shown above.
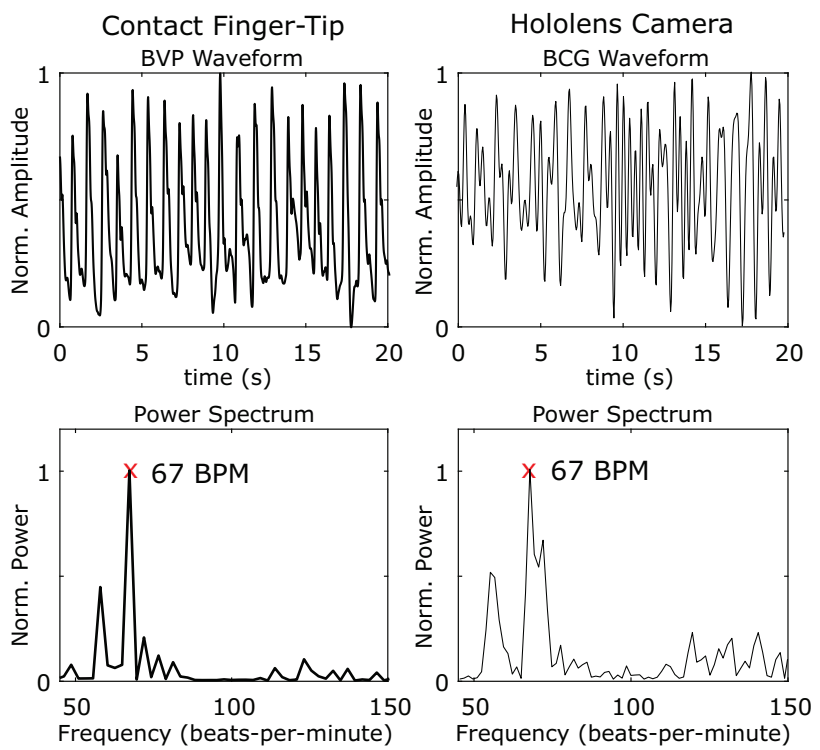

Figure 6: Examples of pulse waveforms from the finger-tip sensor and iBCG measurements. Top) The BCG waveform from the Hololens and the PPG waveform from the fingertip sensor. Bottom) The power spectra of the signals and the detected HR. 
rate is also displayed close to the subject's head. Information is placed onto floating billboards in the holographic space, see Figure 3. Screenshots of the visualization captured through Cardiolens are shown in Figure 4.

The heart rate of the wearer is displayed in the top right-hand corner of the display. This information is displayed relative to the wearer and is not tied to a location in the real-world. Finally, an additional semi-transparent mesh can be applied in the wearers field of view to illustrate their heart rate. A sketch of the resulting visual interface can be seen in Figure 2.

\section{VALIDATION OF PHYSIOLOGICAL MEASUREMENTS}

We collected one-minute recordings from 11 people ( 3 females) with a range of skin tones (east Asian, south Asian, white) using the Cardiolens system alongside gold-standard contact BVP measurements. Some participants were wearing make-up and others had facial hair, neither of these factors were deletarious to the results. Gold standard measurements were obtained from the index finger of the non-dominant hand using a Nonin Onyx II 9560 digital finger-tip pulse oximeter. The BVP waveform was streamed via Bluetooth and logged on a laptop computer. The Cardiolens recordings and finger-tip PPG measurements were synchronized by using the same computer to log and store the data. For validation purposes we stored the recorded videos. The pulse oximeter data was sampled at $75 \mathrm{~Hz}$ and the videos were stored at 24 frames-per-second $(\mathrm{Hz})$ and at $\mathrm{HD}$ resolution.

The participants completed two tasks each of one-minute. During the tasks the participant was asked to not move around or speak but other than that their motions were not restricted. The recordings were captured indoors in a space that combined artificial and natural light. No dedicated light sources were used for illumination, only those normally present in the environment. Thumbnail example frames from one of the recordings can be seen in Figure 5.

\subsection{Tasks}

4.1.1 Imaging Photoplethysmography. We recorded the face of the participant using the Hololens camera, worn by an experimenter, for one minute. The participant and the experimenter were seated one meter apart during the trial. The participants were asked to relax but their head motions and facial expressions were not restricted.

4.1.2 Imaging Ballistocardiography. During this task the participant wore the Hololens and was asked to sit for one minute. We placed a reference grid in the field-of-view of the camera so that there were edges/points that could be tracked for recovering the motion trajectories. The reference grid was placed at one meter from the participant.

\subsection{Results}

To evaluate accuracy we compared the Cardiolens measurements for each task with the gold-standard contact measurements. We computed the accuracy after dividing the data into non-overlapping windows. We used three evaluation metrics: the median absolute error, mean absolute error and the root mean squared error (RMSE) between the heart rate estimates and the gold-standard. For the iPPG measurements we evaluated three window sizes 20,30 and 60 seconds. For the 11 participants there were 1160 s windows, $2230 \mathrm{~s}$ windows and 3320 s windows. Due to the sensitive nature of the iBCG measurements, we used only a 60 s window for recovering the BCG signal, thus there were 1160 s windows.

4.2.1 Imaging Photoplethysmography. Heart Rate: Table 1 shows the absolute error and RMSE between the PPG measurements and the contact finger-tip pulse oximeter. The mean absolute error was 2.34, 1.62 and 1.85 BPM for window sizes of 20,30 and 60 s respectively. This suggests that a 60 s window does not offer a performance benefit versus a $30 \mathrm{~s}$ window. The median error for a $30 \mathrm{~s}$ window was $0.12 \mathrm{PBM}$. With a range of heart rates in our PPG dataset from $48 \mathrm{BPM}$ to $76 \mathrm{BPM}$ these errors show that heart rate detection via iPPG from the head worn device is very accurate.

Figure 5 shows examples of the detected waveforms from the contact sensor and the camera, alongside the power spectrum for each signal. Very little frequency domain information is lost by the camera. Figure 7 shows the correlation $(r=0.9, p<0.01)$ between the measurements for the 30 second window size.

Heart Rate Variability: Using a peak detection algorithm we identified the peaks within the iPPG and gold-standard waveforms. These were used to calculate inter-beat intervals (IBIs), this is the inverse of the instantaneous heart rate. Using the Lomb-Scargle method we calculated the HRV power spectrum which is useful for estimating parasympathetic (rest-digest) and sympathetic (fightflight) responses. Figure 8 shows the IBIs and HRV power spectra for two participants. Power in the $0.15-0.4 \mathrm{~Hz}$ range of the HRV power spectrum is connected to respiratory sinus arrhythmia (RSA), a cardiorespiratory phenomenon characterized by fluctuations in the timing heart beats that are in phase with inhalation and exhalation. Dominant RSA peaks were observed in a majority of the power spectra; however, we do not attempt to rigorously validate respiration rate results here.

While these are preliminary results they show promising evidence that we can recover subtle changes in physiological state using the head-worn device and visualize them in close-to real-time. This opens the possibility to augment the world with a representation of the level stress an individual is experiencing [McDuff et al. 2016].
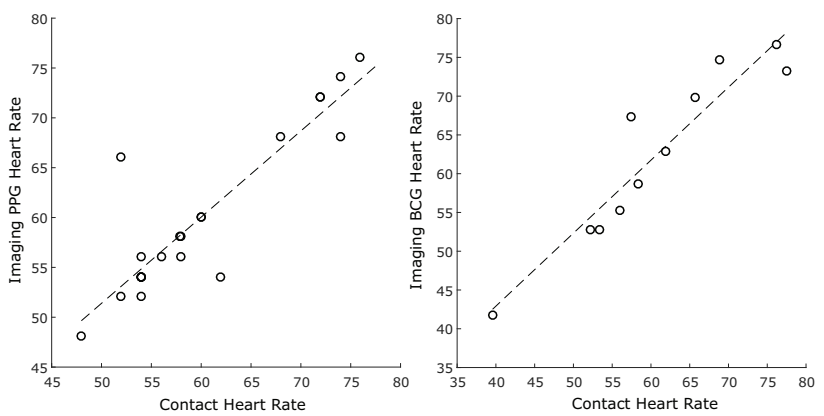

Figure 7: Scatter plots of the heart rate estimates from iPPG and iBCG measurements against the gold-standard contact measurements. The heart rates ranged from 40 to $80 \mathrm{BPM}$. 
Table 1: The absolute error and root mean squared error in heart rate estimates between the contact finger-tip measurements from the pulse oximeter and the Cardiolens system. We compare results using a $20 \mathrm{~s}, 30 \mathrm{~s}$ and $60 \mathrm{~s}$ window sizes for iPPG measurement. We use a 60 s window for iBCG measurement.

\begin{tabular}{cccc} 
& & \multicolumn{2}{c}{ HR Error (BPM) } \\
Error & Win. Size (s) & iPPG & iBCG \\
\hline \hline Abs. Median & & 0.21 & - \\
Abs. Mean & \multirow{2}{*}{20} & 2.34 & - \\
RMSE & & 4.24 & - \\
\hline Abs. Median & \multirow{2}{*}{30} & 0.12 & - \\
Abs. Mean & \multirow{2}{*}{1.62} & - \\
RMSE & & 3.74 & - \\
\hline Abs. Median & & 0.06 & 0.97 \\
Abs. Mean & \multirow{2}{*}{60} & 1.85 & 2.71 \\
RMSE & & 3.90 & 3.98 \\
\hline
\end{tabular}

4.2.2 Imaging Ballistocardiography. Heart Rate: Table 1 shows the absolute error and RMSE between the iBCG measurements and the contact finger-tip pulse oximeter. The absolute error is under 3 BPM, which at an average heart rate is only about $4 \%$ error.

Figure 6 shows an example of detected BCG waveform and the corresponding power spectra. It is clear that the signal captures much of the frequency information of the pulse wave. Figure 7 shows the correlation $(r=0.9, p<0.01)$ between the measurements for the 60 second window size.

Overall, the results show that using the head-mounted camera of the mixed-reality device we can accurately capture the pulse signal and heart rate of the partner and the wearer, using iPPG and iBCG methods respectively.

Heart Rate Variability: As with the iPPG analysis we attempted to recover the inter-beat intervals from the BCG signals; however, it was much more challenging given the iBCG waveforms. Although the dominant heart rate frequency was identifiable the individual peaks were not. Using accelerometer or gyroscope data might lead to waveforms with more easily identifiable peaks.

\subsection{Qualitative Results}

We experimented with several variations of the mixed reality visualization. We tried displaying the heart rate as a number, the pulsatile skin augmentation and showing the pulse waveform itself for the most recent 20 second window of analysis. Several individuals noted that viewing the waveform was not useful and was rather "distracting". The skin augmentation was very intuitive; however, it was difficult to interpret whether the pulsing was fast or slow in absolute terms. Therefore, having the heart rate number was a useful aid. Furthermore, some people felt they could judge whether the measurements were accurate give their own knowledge of their resting heart rate. For some applications simply seeing the pulse information may not be enough. However, the participants noted that if it was possible to visualize a measure of "stress" based on the variability of the heart beats that may be very informative.
Several participants commented on the ethical issues presented by a device that could allow someone to "see" such private information in near real-time. They mentioned that this might effect how they interacted with the other person. They reasoned that unlike with facial expressions they could not control their physiological responses and thus it might be particularly invasive. Humans have created social norms around facial expressions and use facial controls as a tool in social contexts, the same does not exist for physiology.

\section{DISCUSSION}

The ability to measure and visualize the physiological signals of ourselves and those around us in real-time presents numerous new opportunities for augmented reality and virtual reality (VR) applications. Cardiolens can be applied to any stereoscopic head-mounted display with a camera. The system allows for hands-free interactions while augmenting the natural scenes with physiological and affective information. In this section we discuss the implications of integrating Cardiolens into current devices.

\subsection{Immersive Experiences}

Immersive experiences (such as those possible with mixed-reality devices) are likely to lead to more intense affective and emotional responses than traditional computer interactions [Gonzalez-Franco and Lanier 2017; González-Franco et al. 2014; Sanchez-Vives and Slater 2005]. These emotions can be measured through physiological signals [Meehan et al. 2002; Padrao et al. 2016; Spanlang et al. 2014]. Importantly, monitoring the physiological state of the wearer and those around them in a non-invasive way could help indicate undesirable effects, such as simulator sickness [Smolyanskiy and Gonzalez-Franco 2017]. For example, the system could recommend participants take a break from the device when they exceed certain physiological thresholds. Eventually, presenting this information could not only help participants avoid nauseating effects but also train them with coping mechanisms.

\subsection{Social Interactions}

Cardiolens can be used to increase the interoceptive awareness of the user during social interactions. Having a device that can provide this information in real-time, such as Cardiolens, would help augment the physiological states of both the user and the partner/interlocutor. This augmentation would build interoceptive awareness which is known to increase the intensity of emotional responses [Critchley et al. 2004].

The system could help people with impaired social skills, or suffering from autism, increase awareness of their physiological state and thus adapt their social behavior. Arguably the same would be true for socially-able users. Indeed, social performance has long been linked to unconscious mimicry, which is most directly linked to the mirror neuron system [Rizzolatti and Craighero 2004]. Humans automatically enable these resonance mechanisms to reduce out-group biases [Lakin et al. 2008]. Interestingly, the mimicry goes far beyond behavioral and speech patterns and humans unconsciously change their own physiological interoceptive functioning to match others in many aspects in life. 


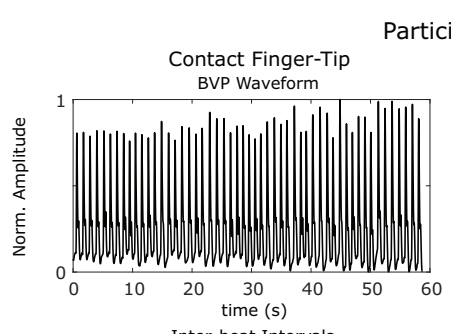

Participant A
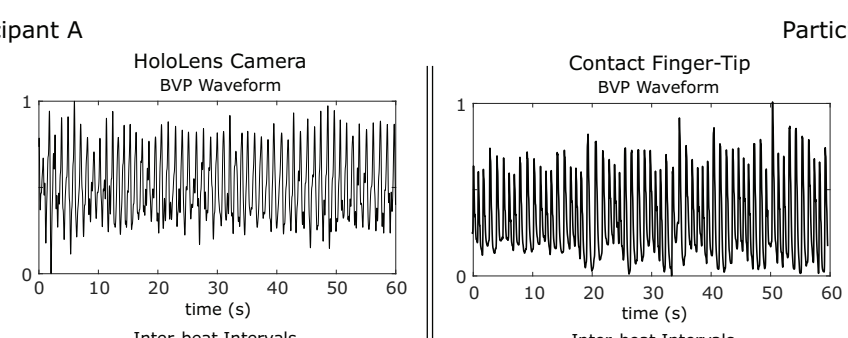

Participant B
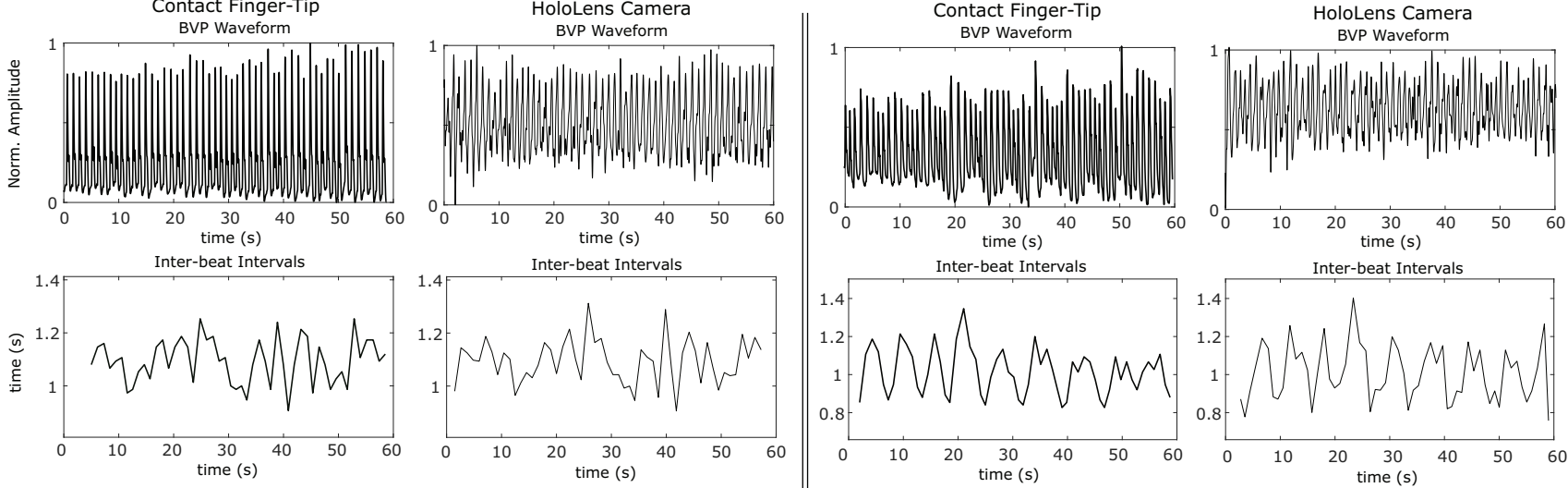

HRV Power Spectrum
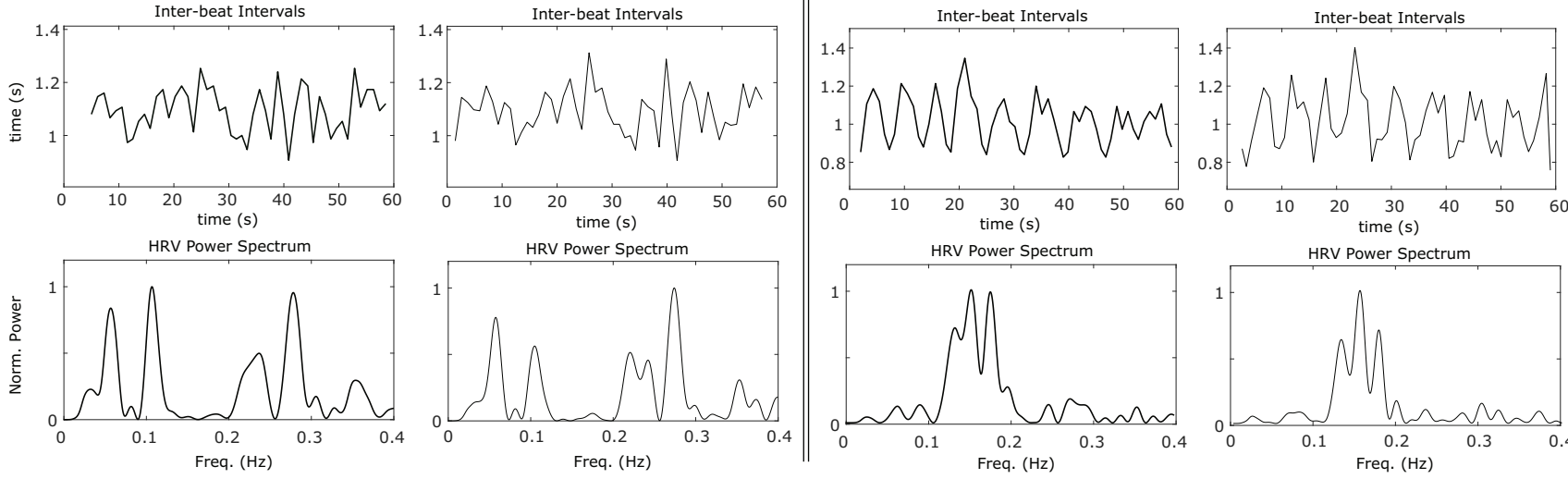

HRV Power Spectrum

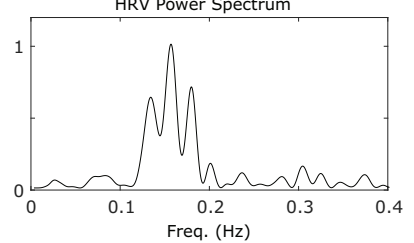

Figure 8: The pulse wave (top), inter-beat intervals (middle) and HRV power spectra (bottom) for two participants. Comparisons between the contact sensor (thick line) and iPPG (thin line) signals are shown in each case. The inter-beat intervals (1/instantaneous heart rate) measurements are quite accurate and this allows us to recover the HRV power spectrum which is useful for estimating sympathetic and parasympathetic activation and respiration rate. For the example on the right the respiratory sinus arrhythmia (RSA) is clearly present in the IBI signal.

Emotional contagion (e.g. stress or anger passing from a mother to a child) is one example of physiological synchrony between people. In other cases, the process can be purely physiological and independent of the original emotions. Synchronization might take place over long exposures to others (e.g. menstrual synchronization [McClintock 1971]), but also during short exposures (e.g. respiration rate and/or heart rate synchronization of interlocutors during conversations [Sowden et al. 2016].)

There are beneficial effects of this interoceptive matching, as when synchronous physiological signals are achieved, participants are more likely to pass a job interview. On the contrary decreased interoceptive awareness has been shown to follow social exclusion [Durlik and Tsakiris 2015]. Cardiolens could be used to both learn more about how awareness might impact these mimicry effects, and potentially feed that information into real-life interactions to help boost synchrony between pairs.

\subsection{Skilled Observer}

In many situations there might not be social interaction but still an observer might benefit from knowing physiological variables before, during, and after a critical activity. There are many scenarios in which observers currently rely on their own intuition; however, explicit knowledge of affective and physiological signals could help develop more objective decision-making.

In sports, trainers could evaluate athletes at a simple gaze or during a routine. On a more trivial note, it could be used to augment the performance of players during gambling sessions. We foresee that as camera technology continues to develop it will be possible to visualize subtle changes in blood perfusion across the body. This could aid surgeons interested in knowing whether a transplanted organ/tissue has blood flowing to it. These and other tasks could benefit from a system such as Cardiolens. Further research on image processing could find particular patterns that reveal blood oxygenation, body temperature or intoxication with chemical components. Thus making more information explicit for skilled observers to help them with objective decision-making.

\subsection{Ethics and Privacy}

From an ethical perspective there are some aspects that need to be addressed given the potential surveillance applications of this system. There is a natural and well studied conflict between privacy and the advancement of technology in society [Westin 1968]. In this regard, Cardiolens presents a non-invasive and non-explicit system able to retrieve physiological parameters from anybody just by looking at them. So far the discussion of the implications has focused on enabling positive scenarios. However, there are negative applications of this technology that would infringe on data privacy rights [Slobogin 2002].

When applied in-situ, this technology might be able to perform non-explicit monitoring of people who have not consented and are not aware of being recorded. We see a necessity to develop an antidote, a way to prevent this monitoring. There is a need for a system that hinders heart beat retrieval. Based on our initial investigations, random IR light patterns projected on to the skin will prevent the 
retrieval of a correct heart beat. One could imagine using an IR LED that lights up the user's skin with varying intensities. In that case, our system might confuse the LED signal with the actual heart beat and retrieve inaccurate information. We propose IR, precisely because it will be invisible to the unaided eye, although other types of illuminations would also result in interference.

Without appropriate consent people might start perceiving an otherwise positive device as a mere tool for surveillance. Therefore, besides the ability to block or interfere with monitoring, we also see a need to make explicit when this technology is recording data and if/when it is storing the data. Recently devices, such as Google glass or Snapchat's spectacles, have miniaturized cameras to a point that they are no longer obvious. Hololens provides an LED that indicates when the camera is recording. With time, people will learn that they are being monitored. And in many cases, they will not care. We are already being surveilled at massive scales with CCTV cameras and more recently with microphones. However, it is clear that social norms need to exist around the acceptable use of such technology. The concern would only increase further if Cardiolens also incorporated facial identification technology.

A secondary ethical question that stands out is related to the clear asymmetry in information power produced when this system is worn by one person but not others. The societal divisions between those who can afford technology and those who cannot might cause social problems. However, in general we are very optimistic that if the proposed consent mechanisms are implemented this technology will have more positive than negative applications.

\subsection{Feasibility}

In our validation study we used the Microsoft Hololens and implemented the system in C\#. All the processing is performed on the device and images captured using the front-facing camera. Thus the device does not need to be tethered to a computer, nor does the hardware require any custom adaptation. The results revealed good performance in physiological measurement despite motion of the camera and the head.

Nevertheless, the device suffers from some limitations. Some of these are inerrant to non-contact measurement of physiological signals, and others result from current technological restrictions of the device. The camera is sensitive to abrupt changes of lighting conditions. Which means, the video processing takes 20 seconds to retrieve a pulse waveform.

The holographic overlay layer that displays the subjects pulsating skin is limited by the device. This layer has to be accurately positioned on top of a face. Since Hololens operates in the 3D space and uses stereoscopic vision, this overlay has to be at the same depth as the partner's face. However, if the depth map of the environment is limited or inaccessible the overlay is not well displayed. To address this issue, in our demonstration we estimate the depth from the vertical height of the captured face: a smaller height means that the partner is further away; thus we render the overlay at a larger depth. This technique is sufficient to present the information in real-time but could be more accurate. In the future, the device itself may give direct assess to the captured depth map.

\section{CONCLUSIONS}

Cardiolens is the first mixed reality system that enables real-time, hands-free measurement and visualization of physiological signals. The system uses images from the front-facing camera to recover the vital signs of both the wearer and the person they are looking at simultaneously. We presented the technical implementation of the system and describe the mixed reality interface that showcases realtime visualization of normally "hidden" physiological parameters.

We validated the system by capturing measurements of 11 subjects using Hololens alongside gold-standard contact signals. The results show accurate HR rate measurement of both the wearer and a person they are looking at. We achieved a mean absolute error below 3 BPM for both iPPG and iBCG measurements. We also presented baseline results for heart rate variability measurement using the iPPG signal. Qualitative feedback revealed participants were intrigued by the ability to "see" these hidden signals and that numerical representation of the heart rate and skin augmentation were more effective than a display of the pulse waveform itself.

We discussed the implications of our system and presented novel visualizations of the physiological measurements in the real-world. Users found the ability to magnify the blood volume pulse signal by augmenting the appearance of the skin particularly powerful, some refereed to it as having a "super power".

\section{ACKNOWLEDGEMENTS}

The authors acknowledge the support of the French National Agency for Research (Agence Nationale de la Recherche ANR) under the grant ANR-14-CE24-0006-01 project "TERANOVA" and the SESAR Research and Innovation Action Horizon 2020 under project "MOTO" (The embodied reMOte TOwer).

\section{REFERENCES}

John Allen. 2007. Photoplethysmography and its application in clinical physiological measurement. Physiological measurement 28, 3 (2007), R1.

Antoine Bechara and Nasir Naqvi. 2004. Listening to your heart: interoceptive awareness as a gateway to feeling. Nature neuroscience 7, 2 (2004), 102-103.

Hugo D Critchley, Stefan Wiens, Pia Rotshtein, Arne Öhman, and Raymond J Dolan. 2004. Neural systems supporting interoceptive awareness. Nature neuroscience 7, 2 (2004), 189-195.

David Da He, Eric S Winokur, and Charles G Sodini. 2012. An ear-worn continuous ballistocardiogram (BCG) sensor for cardiovascular monitoring. In Engineering in Medicine and Biology Society (EMBC), 2012 Annual International Conference of the IEEE. IEEE, 5030-5033.

Caroline Durlik and Manos Tsakiris. 2015. Decreased interoceptive accuracy following social exclusion. International fournal of Psychophysiology 96, 1 (2015), 57-63.

Shakith Fernando, Wenjin Wang, Ihor Kirenko, Gerard de Haan, Sidarto Bambang Oetomo, Henk Corporaal, and Jan van Dalfsen. 2015. Feasibility of contactless pulse rate monitoring of neonates using google glass. In Proceedings of the 5th EAI International Conference on Wireless Mobile Communication and Healthcare. ICST (Institute for Computer Sciences, Social-Informatics and Telecommunications Engineering), EAI, 198-201.

Mar Gonzalez-Franco and Jaron Lanier. 2017. Model of Illusions and Virtual Reality. Frontiers in psychology 8 (2017).

Mar González-Franco, Tabitha C Peck, Antoni Rodríguez-Fornells, and Mel Slater. 2014. A threat to a virtual hand elicits motor cortex activation. Experimental brain research 232, 3 (mar 2014), 875-87.

Javier Hernandez, Yin Li, James M Rehg, and Rosalind W Picard. 2014. Bioglass: Physiological parameter estimation using a head-mounted wearable device. In Wireless Mobile Communication and Healthcare (Mobihealth), 2014 EAI 4th International Conference on. IEEE, 55-58.

Javier Hernandez, Daniel McDuff, and Rosalind W Picard. 2015. Biowatch: estimation of heart and breathing rates from wrist motions. In Proceedings of the 9th International Conference on Pervasive Computing Technologies for Healthcare. ICST (Institute for Computer Sciences, Social-Informatics and Telecommunications Engineering), 169-176. 
Jessica L Lakin, Tanya L Chartrand, and Robert M Arkin. 2008. I am too just like you: Nonconscious mimicry as an automatic behavioral response to social exclusion. Psychological science 19, 8 (2008), 816-822.

Martha K McClintock. 1971. Menstrual synchrony and suppression. Nature (1971).

Daniel McDuff, Justin R Estepp, Alyssa M Piasecki, and Ethan B Blackford. 2015. A survey of remote optical photoplethysmographic imaging methods. In 2015 37th Annual International Conference of the IEEE Engineering in Medicine and Biology Society (EMBC). IEEE, 6398-6404

Daniel McDuff, Sarah Gontarek, and Rosalind W Picard. 2014. Remote detection of photoplethysmographic systolic and diastolic peaks using a digital camera. IEEE Transactions on Biomedical Engineering 61, 12 (2014), 2948-2954.

Daniel McDuff, Javier Hernandez, Sarah Gontarek, and Rosalind W Picard. 2016. COGCAM: Contact-free Measurement of Cognitive Stress During Computer Tasks with a Digital Camera. In Proceedings of the 2016 CHI Conference on Human Factors in Computing Systems. ACM, 4000-4004.

Michael Meehan, Brent Insko, Mary Whitton, and Frederick P Brooks Jr. 2002. Physiological measures of presence in stressful virtual environments. ACM Transactions on Graphics (TOG) 21, 3 (2002), 645-652.

Gonçalo Padrao, Mar Gonzalez-Franco, Maria V Sanchez-Vives, Mel Slater, and Antoni Rodriguez-Fornells. 2016. Violating body movement semantics: Neural signatures of self-generated and external-generated errors. Neuroimage 124 (2016), 147-156.

Ming-Zher Poh, Daniel McDuff, and Rosalind W Picard. 2010. Non-contact, automated cardiac pulse measurements using video imaging and blind source separation. Optics Express 18, 10 (2010), 10762-10774.

Ming-Zher Poh, Daniel McDuff, and Rosalind W Picard. 2011. Advancements in noncontact, multiparameter physiological measurements using a webcam. IEEE Transactions on Biomedical Engineering 58, 1 (2011), 7-11.

Giacomo Rizzolatti and Laila Craighero. 2004. The mirror-neuron system. Annu. Rev. Neurosci. 27 (2004), 169-192.

Maria V Sanchez-Vives and Mel Slater. 2005. From presence to consciousness through virtual reality. Nature Reviews Neuroscience 6, 4 (2005), 332-339.

Dangdang Shao, Yuting Yang, Chenbin Liu, Francis Tsow, Hui Yu, and Nongjian Tao. 2014. Noncontact monitoring breathing pattern, exhalation flow rate and pulse transit time. IEEE Transactions on Biomedical Engineering 61, 11 (2014), 2760-2767.

Christopher Slobogin. 2002. Public privacy: camera surveillance of public places and the right to anonymity. Miss. lf 72 (2002), 213.
Nikolai Smolyanskiy and Mar Gonzalez-Franco. 2017. Stereoscopic First Person View System for Drone Navigation. Frontiers in Robotics and AI 4 (2017), 11.

Sophie Sowden, Rebecca Brewer, Caroline Catmur, and Geoffrey Bird. 2016. The specificity of the link between alexithymia, interoception, and imitation. fournal of Experimental Psychology: Human Perception and Performance 42, 11 (2016), 1687.

Bernhard Spanlang, Jean-Marie Normand, David Borland, Konstantina Kilteni, Elias Giannopoulos, Ausiàs Pomés, Mar González-Franco, Daniel Perez-Marcos, Jorge Arroyo-Palacios, Xavi Navarro Muncunill, et al. 2014. How to build an embodiment lab: achieving body representation illusions in virtual reality. Frontiers in Robotics and $A I 1$ (2014), 9.

Isaac Starr, AJ Rawson, HA Schroeder, and NR Joseph. 1939. Studies on the estimation of cardiac ouptut in man, and of abnormalities in cardiac function, from the heart's recoil and the blood's impacts; the ballistocardiogram. American fournal of Physiology-Legacy Content 127, 1 (1939), 1-28.

Paul Viola and Michael Jones. 2001. Rapid object detection using a boosted cascade of simple features. In Computer Vision and Pattern Recognition, 2001. CVPR 2001. Proceedings of the 2001 IEEE Computer Society Conference on, Vol. 1. IEEE, I-511.

Neal Wadhwa, Michael Rubinstein, Frédo Durand, and William T Freeman. 2014. Riesz pyramids for fast phase-based video magnification. In Computational Photography (ICCP), 2014 IEEE International Conference on. IEEE, 1-10.

Wenjin Wang, Albertus C den Brinker, Sander Stuijk, and Gerard de Haan. 2017. Color-Distortion Filtering for Remote Photoplethysmography. In Automatic Face \& Gesture Recognition (FG 2017), 2017 12th IEEE International Conference on. IEEE, 71-78.

W Wang, S Stuijk, and G de Haan. 2015. Exploiting Spatial Redundancy of Image Sensor for Motion Robust rPPG. IEEE Transactions on Biomedical Engineering 62, 2 (2015), 415-425.

Alan F Westin. 1968. Privacy and freedom. Washington and Lee Law Review 25, 1 (1968), 166

Hao-Yu Wu, Michael Rubinstein, Eugene Shih, John V Guttag, Frédo Durand, and William T Freeman. 2012. Eulerian video magnification for revealing subtle changes in the world. ACM Trans. Graph. 31, 4 (2012), 65.

Oi Zhang Oingtian Wu, Yimin Zhou, Xinyu Wu, Yongsheng Ou, and Huazhang Zhou. 2017. Webcam-based, non-contact, real-time measurement for the physiological parameters of drivers. Measurement 100 (2017), 311-321. 